\title{
CORRECTION
}

\section{Correction: Neonatal acute kidney injury risk stratification score: STARZ study}

Sanjay Wazir, Sidharth Kumar Sethi, Gopal Agarwal, Abhishek Tibrewal, Rohan Dhir, Naveen Bajaj, Naveen Parkash Gupta, Shishir Mirgunde, Jagdish Sahoo, Binesh Balachandran, Kamran Afzal, Anubha Shrivastava, Jyoti Bagla, Sushma Krishnegowda, Ananth Konapur, Kritika Soni, Abhyuday Rana, Timothy Bunchman and Rupesh Raina

(๑) The Author(s), under exclusive licence to the International Pediatric Research Foundation, Inc 2021

Pediatric Research (2022) 91:1302-1304; https://doi.org/10.1038/s41390-021-01674-5

Correction to: Pediatric Research https://doi.org/10.1038/s41390021-01573-9, published online 19 May 2021

Unfortunately, several errors occurred in Tables 1 and 3, Fig. 5 and the "Discussion" section. The corrected Tables 1 and 3 and Fig. 5 are given below. In the "Discussion" section the information on urine output has been changed to $<1.32 \mathrm{ml} / \mathrm{kg} / \mathrm{h}$. The original article has been corrected. 
Table 1. The significant variables in the best-fit model identified based on the multivariate logistic regression technique with the stepwise backward elimination method [ $n=310 ; 31$ independent variables].

\section{Variables}

Age at entry in $\mathrm{NICU}^{\mathrm{a}}(<25.5$; Ref.: $\geq 25.5 \mathrm{~h})$

PPV in the delivery room (Yes; Ref.: No)

Gestational age ( $<28$; Ref.: $\geq 28$ weeks)

Sepsis (during the NICU stay) (Yes; Ref.: No)

Significant cardiac disease (Yes; Ref.: No)

Urine output $^{\mathrm{a}, \mathrm{b}}(<1.32$; Ref.: $\geq 1.32 \mathrm{ml} / \mathrm{kg} / \mathrm{h}$ )

Serum creatinine ${ }^{\mathrm{a}, \mathrm{b}}(\geq 0.98$; Ref.: $<0.98 \mathrm{mg} / \mathrm{dl}$ )

Use of nephrotoxic drugs (Yes; Ref.: No)

Use of furosemide (Yes; Ref.: No)

Use of inotrope(s) (Yes; Ref.: No)

\begin{tabular}{l}
$\boldsymbol{\beta}$ coefficient \\
1.29 \\
1.66 \\
1.51 \\
1.46 \\
\hline 2.16 \\
1.59 \\
\hline 4.60 \\
\hline 2.46 \\
1.96 \\
\hline 3.78 \\
\hline
\end{tabular}

Adjusted RR (95\% Cl)

$p$ value

$2.06(1.05-2.97)$

0.04

$1.91(1.05-2.32)$

0.04

$2.01(1.02-2.74)$

0.02

$1.98(1.04-2.54)$

0.04

$2.81(1.06-3.56)$

0.04

$2.29(1.21-3.01)$

0.01

$13.44(8.44-15)$

$<0.001$

$2.76(1.6-3.19)$

0.004

$2.39(1.06-2.98)$

$2.79(1.01-2.91)$ Adjusted for all the variables with $p<0.05$ in the univariate analysis. Enteral fluid intake and serum urea were not considered in the multivariate analysis due to missing data in $>50 \%$ of the patients.

PPV positive pressure ventilation, Ref. reference, NICU neonatal intensive care unit.

${ }^{a}$ The continuous variables observed to be independently associated with AKI in Mann-Whitney $U$ test were categorized into two groups based on the cut-off threshold value (sensitivity and specificity is observed to be the highest) identified by ROC analysis.

${ }^{\mathrm{b}}$ First $12 \mathrm{~h}$ post admission in NICU.

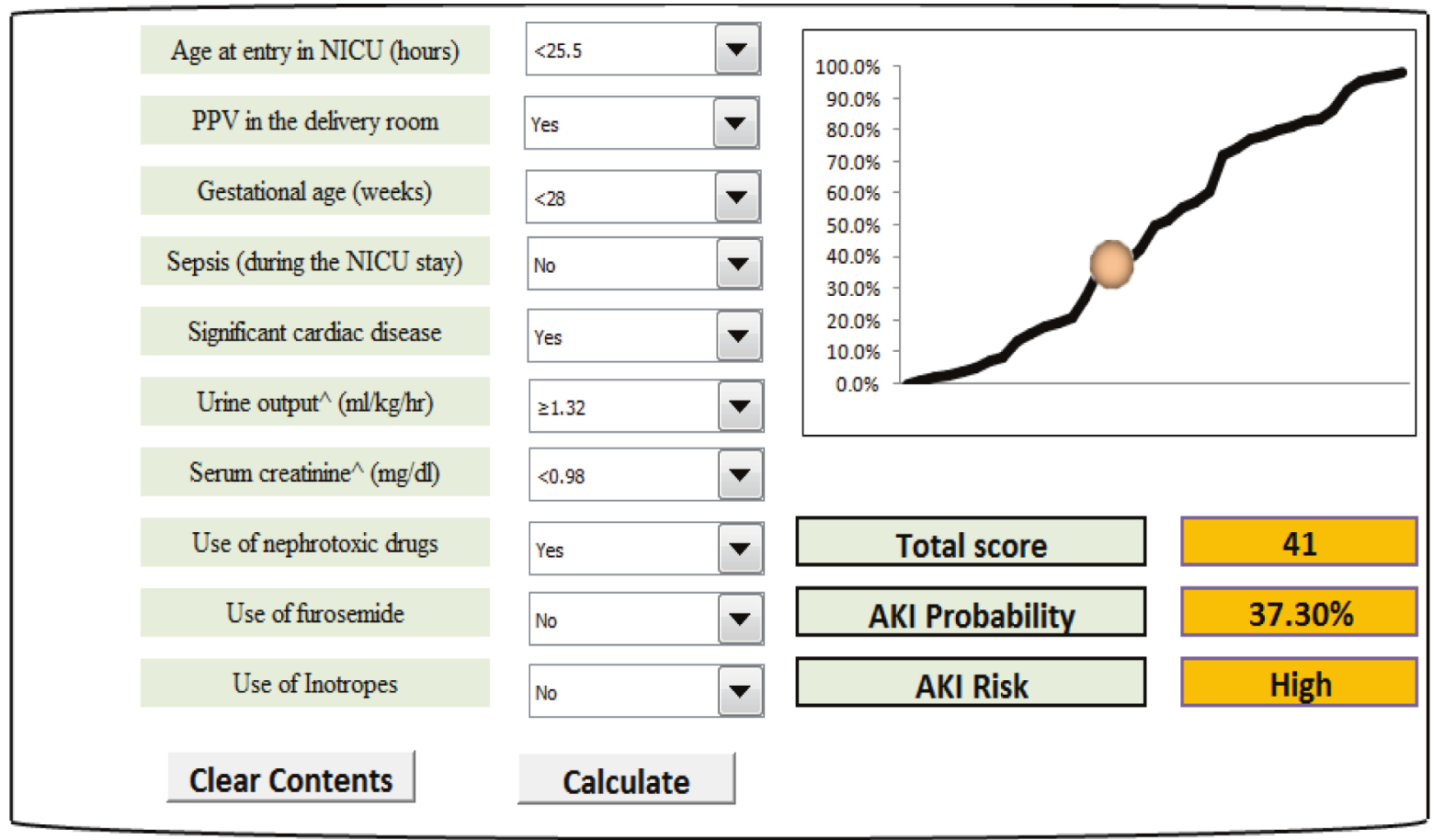

Please click clear contents, update the values of all the variables as per the data of the children, and then click calculate

Fig. 5 Scoring system dashboard. The scoring system considers variable such as age at entry in the NICU, PPV in the delivery room, gestational age, presence of sepsis and significant cardiac disease, urine output, serum creatinine levels, use of nephrotoxic drugs, furosemide, and inotropes to calculate AKI probability and risk. ${ }^{\wedge}$ First $12 \mathrm{~h}$ post admission in NICU. 
Table 3. Score assigned to each significant variable of the best-fit model.

\begin{tabular}{|c|c|c|c|c|}
\hline Variables & $\beta$ coefficient & $\beta$ coefficient $\times 10$ & Score to the nearest integer $/ 100$ & Assigned score \\
\hline \multicolumn{5}{|c|}{ Age at entry in $\mathrm{NICU}^{\mathrm{a}}(\mathrm{h})$} \\
\hline$<25.5$ & 1.29 & 12.9 & 6 & 6 \\
\hline$\geq 25.5$ & & & & 0 \\
\hline \multicolumn{5}{|c|}{ PPV in the delivery room } \\
\hline Yes & 1.66 & 16.6 & 7 & 7 \\
\hline No & & & & 0 \\
\hline$<28$ & 1.51 & 15.1 & 7 & 7 \\
\hline$\geq 28$ & & & & 0 \\
\hline \multicolumn{5}{|c|}{ Sepsis (during the NICU stay) } \\
\hline Yes & 1.46 & 14.6 & 6 & 6 \\
\hline No & & & & 0 \\
\hline$<1.32$ & 1.59 & 15.9 & 7 & 7 \\
\hline$\geq 1.32$ & & & & 0 \\
\hline \multicolumn{5}{|c|}{ Serum creatinine $^{\mathrm{a}}(\mathrm{mg} / \mathrm{dl})$} \\
\hline$\geq 0.98$ & 4.60 & 46.0 & 20 & 20 \\
\hline$<0.98$ & & & & 0 \\
\hline \multicolumn{5}{|c|}{ Use of nephrotoxic drugs } \\
\hline Yes & 2.46 & 24.6 & 11 & 11 \\
\hline No & & & & 0 \\
\hline \multicolumn{5}{|c|}{ Use of furosemide } \\
\hline
\end{tabular}

Nephrotoxic drugs included vancomycin or colistin or amphotericin B.

PPV positive pressure ventilation, NICU neonatal intensive care unit.

${ }^{\mathrm{a}}$ First $12 \mathrm{~h}$ post admission in NICU. 